\title{
Multiscale dynamic wetting of a droplet on a lyophilic pillar-arrayed surface
}

\author{
Quanzi Yuan and Ya-Pu Zhao $\dagger$ \\ State Key Laboratory of Nonlinear Mechanics, Institute of Mechanics, Chinese Academy of Sciences, \\ Beijing 100190, People's Republic of China
}

(Received 31 December 2011; revised 14 September 2012; accepted 31 October 2012)

Dynamic wetting of a droplet on lyophilic pillars was explored using a multiscale combination method of experiments and molecular dynamics simulations. The excess lyophilic area not only provided excess driving force, but also pinned the liquid around the pillars, which kept the moving contact line in a dynamic balance state every period of the pillars. The flow pattern and the flow field of the droplet on the pillar-arrayed surface, influenced by the concerted effect of the liquid-solid interactions and the surface roughness, were revealed from the continuum to the atomic level. Then, the scaling analysis was carried out employing molecular kinetic theory. Controlled by the droplet size, the density of roughness and the pillar height, two extreme regimes were distinguished, i.e. $R \sim t^{1 / 3}$ for the rough surface and $R \sim t^{1 / 7}$ for the smooth surface. The scaling laws were validated by both the experiments and the simulations. Our results may help in understanding the dynamic wetting of a droplet on a pillar-arrayed lyophilic substrate and assisting the future design of pillar-arrayed lyophilic surfaces in practical applications.

Key words: contact lines, drops, micro-/nano-fluid dynamics

\section{Introduction}

Dynamic wetting of a droplet on a lyophilic pillar-arrayed substrate is of significant interest in a broad range of applications, such as biomedicine (Nagrath et al. 2007), microfluidics (Skelley et al. 2009), lab-on-a-chip (Fu, Mao \& Han 2009) and fogharvesting (Parker \& Lawrence 2001). To utilize these phenomena in practical applications (Quéré 2008), it is essential to explore and understand the dynamic wetting behaviours influenced by the combined effect of wetting properties and the surface roughness $r o$, which is the ratio between the actual and projected surface areas. The liquid-solid interactions at the interface become rather complicated (Karniadakis, Beşkök \& Aluru 2005). The forest of pillars seems to introduce obstacles in the flow and increases the active area. In fact, owing to the excess solid surface, the topology enhances the wettability and the flow velocity (McHale et al. 2004), causing the initial lyophilic surface to become superlyophilic. As illustrated in figure $1, r o=1+4 d h / p^{2}$ for pillars, where $d, h$ and $p$ are the pillar size, the pillar height and the period of the pillars. 
(a)

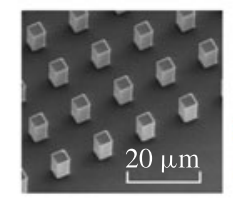

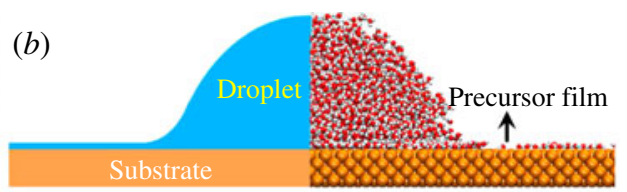

$(c)$
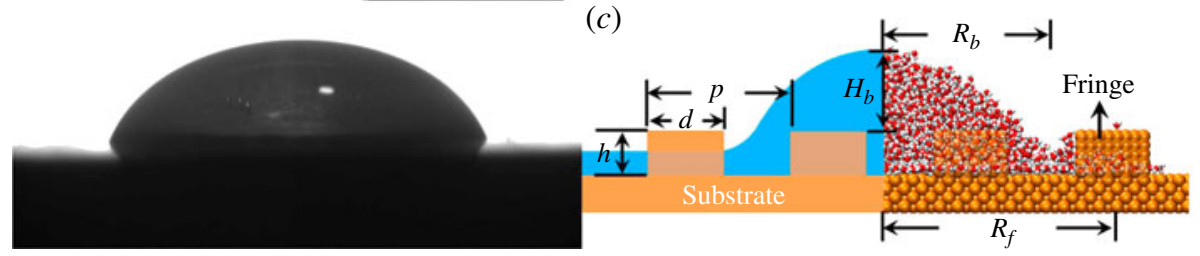

FIgURE 1. (Colour online) (a) Snapshot of a droplet on the pillar-arrayed lyophilic surface. Inset: scanning electron micrograph (SEM) of the pillar arrays. The samples are listed in table 1. Illustration of a droplet wetting $(b)$ on a smooth lyophilic surface, and $(c)$ on a pillar-arrayed lyophilic surface. $R_{b}, H_{b}$ and $R_{f}$ are the radius and height of the bulk droplet, and the average fringe radius, respectively; $d, h$ and $p$ are the pillar size, the pillar height and the period of the pillars, respectively.

The dynamic wetting of a droplet on a lyophilic smooth solid surface has been studied intensely for decades. Since a liquid droplet is much smaller than the capillary length $l_{C A}=\sqrt{\gamma_{L V} / \rho g}\left(\gamma_{L V}, \rho\right.$ and $g$ are the liquid-vapour interfacial tension, liquid density and the acceleration of gravity, respectively), gravity is ignored. Hence, the driving force is the interfacial tension, while energy dissipation occurs around the moving contact line (MCL). There are essentially two theoretical models to interpret the physics of the wetting behaviours (Bonn et al. 2009; Ren, Hu \& E 2010), known as the hydrodynamic model and molecular kinetic theory (MKT).

(i) Within the framework of the hydrodynamic model, three length scales are distinguished (Dussan 1979). The macroscopic region is on the scale of the droplet radius, in which the apparent contact angle $\theta_{a}$ is defined; the mesoscopic region is on the scale of $3 C a R / \theta_{a}$ (the capillary number $C a=\mu U / \gamma_{L V}$, and $\mu, U$ and $R$ are the viscosity, velocity and droplet radius, respectively) around the three-phase zone, in which the interface is bent by the competition between surface tension and viscosity, resulting in a rapid change of interface slope; the microscopic region is on the scale of nanometres around the contact line, where the microscopic contact angle $\theta_{m}$ is governed by the short-range intermolecular forces, i.e. the disjoining pressure (Derjaguin, Churaev \& Muller 1987). By solving the Navier-Stokes equation with no-slip boundary, Tanner (1979) first obtained the scaling law of $R \sim t^{1 / 10}$. However, Huh \& Scriven (1971) noted a paradox and pointed out that the no-slip boundary condition would lead to stress singularity at the MCL. One solution is to use a slip boundary instead (Dussan 1976; Ren \& E 2007). Cox (1986) carried out an asymptotic analysis to relate quasi-static macroscale and microscale via rapid mesoscale viscous bending. The other solution is to take into account nano/microstructures, which are neglected in ideal models, such as the precursor film (PF) proposed by de Gennes (1985) or the roughness of the solid surface (Hocking 1976). These surface structures, i.e. PF and the surface roughness, are of great importance in the dynamic wetting of a droplet on the lyophilic pillar-arrayed surface, as discussed in $\S \S 2$ and 3 . 
(ii) Within the framework of the MKT model, two length scales are distinguished (Blake 2006). On the molecular scale, the forward and backward frequencies of the liquid molecules in the three-phase zone are modified by the driving force; on the macroscale, the motion of the MCL is determined by the statistical dynamics of the molecules. The energy dissipation is caused by the adsorption-desorption of the liquid molecules on the solid surface, and represented by the effective dynamic friction occurred at the MCL. The MKT model is a bottom-up model, obtaining statistic effects based on information from the atomic level. The scaling law for a droplet on a smooth surface is $R \sim t^{1 / 7}$ (Blake 1993), and validated by experiments (Fetzer, Ramiasa \& Ralston 2009). In $\$ 4$ we will discuss MKT in detail and analyse the dynamic wetting of a droplet on the pillar-arrayed surface using MKT.

However, the dynamic wetting of a droplet on a lyophilic pillar-arrayed surface is far from well understood. Previous studies made an effort to determine the scaling law $R_{f} \sim t^{n}$ of the fringe film in the wicking process by employing the continuum theory, where $R_{f}$ and $n$ are the propagation radius and the scaling exponent, respectively. The pioneering work of Hasimoto (1959) estimated the viscous resistance of the pillars to the fluid by idealizing the pillar arrays as infinitely long cylinders. Taking account of both the viscous resistance from the pillars and the substrate, Ishino et al. did an elegant scaling analysis based on the Washburn law to obtain two extreme regimes for short and tall posts, respectively (Ishino et al. 2007). Then, Srivastava et al. (2010) and Xiao, Enright \& Wang (2010) developed this model to be more accurate. However, while these studies all averaged the MCL to be a straight line (two-dimensional problem) or a circle (three-dimensional problem), the MCL is actually a complex curve (Courbin et al. 2007) and requires a multiscale study. Also, the previous studies focused on the wicking process of a fringe film, i.e. a thin liquid film wetting the solid surface, while a droplet is preferred in practical applications. Kim et al. proposed a scaling law of $R_{f} \sim t^{1 / 4}$ in the early stage of a droplet spreading on the pillar array using the hydrodynamic model (Kim et al. 2011), but the results could not degenerate to a droplet on a smooth surface when ro approaches 1 . There is a lack of a systematic and multiscale study of the dynamic wetting of a droplet on the rough surface.

The dynamic wetting of a droplet on lyophilic pillars is essentially a multiscale process. When a droplet is deposited on a lyophilic pillar array, the fringe penetrates into the space among the pillars, while the bulk water spreads on the base of the fringe. At the nanoscopic level as shown in figure $1(b)$, a thin liquid precursor film propagating ahead of the nominal contact line is a crucial part of the dynamic wetting process (de Gennes 1985; Teletzke, Davis \& Scriven 1987; Yuan \& Zhao 2010). Within the forest of lyophilic pillars, driven by the microscopic disjoining pressure, the PF propagates rapidly and forms a liquid molecular layer on the pillar surface. At the microscopic level, driven by the hydrodynamic pressure, the fringe advances on the base of the PF and fills the space among the pillars, as shown in figure 1(c). On one hand, the grooves between the pillars provide excess driving force to the PF and the fringe (Yuan \& Zhao 2012); on the other hand, the pillars bring extra potential barriers to the PF and excess resistance to the fringe. How could these two opposite effects brought by the pillars achieve a balance in the fast advancing MCL? On the macroscopic level, the radius expansion of the droplet could be observed and measured when the liquid super-wets the surface. To understand the complex liquid-solid interactions of a droplet on a pillar-arrayed lyophilic surface from the atomic to the continuum level, a multiscale study is required. 


$\begin{array}{lcccccc}\text { Sample } & 1 & 2 & 3 & 4 & 5 & 6 \\ d(\mu \mathrm{m}) & 11.66 & 8.84 & 20.29 & 9.11 & 8.82 & 9.18 \\ p(\mu \mathrm{m}) & 17.71 & 15.69 & 26.56 & 20.0 & 24.7 & 29.94 \\ h(\mu \mathrm{m}) & 10.18 & 10.35 & 10.18 & 10.35 & 10.35 & 10.35 \\ \phi_{s}=\frac{d^{2}}{p^{2}} & 0.4335 & 0.3174 & 0.5836 & 0.2075 & 0.1275 & 0.0940 \\ r o=1+\frac{4 d h}{p^{2}} & 2.51 & 2.49 & 2.17 & 1.94 & 1.60 & 1.42\end{array}$

TABLE 1. Substrates of with different roughness used in the experiments.

In this article, the dynamic wetting process of a non-volatile droplet on lyophilic pillars was studied employing multiscale experiments and molecular dynamic (MD) simulations. Under a low-magnification microscope, the MCL seemed to be smooth and circular. Under a high-magnification microscope and a high-speed camera, the details of the MCL were revealed. The pillars accelerated the slow flow, while also resisting or pinning the fast flow. To reach a balance in the propagating velocity, the fringe advanced in a complex pattern repeated during every period of the pillars. The flow pattern and the flow field of the droplet on the pillar-arrayed surface, influenced by the combined effect of the liquid-solid interactions and the surface roughness, were revealed from the continuum (experiments) to the atomic level (MD simulations). Based on the results from the multiscale experiments and MD simulations, the dynamic process and the physical mechanisms were analysed using molecular kinetic theory (MKT). Controlled by the droplet size, the density of roughness and the pillar height, the scaling laws for two regimes were obtained: $R \sim t^{1 / 3}$ for the rough surface and $R \sim t^{1 / 7}$ for the smooth surface. Both the experiments and the simulations validated the scaling laws from MKT. Our results may help in understanding the dynamic wetting of the droplet on a pillar-arrayed lyophilic substrate and assisting the future design of pillar-arrayed lyophilic surfaces in practical applications.

\section{Multiscale experiments}

The micropillar arrays were fabricated on a silicon wafer at the Institute of Microelectronics, Peking University. The Si (100) surface was etched using conventional photolithography followed by a deep reactive ion etching process. The inset in figure 1(a) shows the pillar-arrayed surface (sample 5, table 1). The topological parameters $[d, p, h]$ for experimental samples were varied, as listed in table 1. Since the fringe of the droplet is a thin liquid film, the evaporation of the liquid would greatly affect the wetting process. Hence the liquid was chosen to be non-volatile silicone oil, so that the evaporation effect on the wetting process could be eliminated. We used silicone oil with density $\rho=980 \mathrm{~kg} \mathrm{~m}^{-3}$, viscosity $\mu=0.091 \mathrm{~Pa} \mathrm{~s}$ and surface tension $\gamma_{L V}=0.021 \mathrm{~N} \mathrm{~m}^{-1}$, which completely wets the smooth silicon surface. According to Wenzel's relation (Wenzel 1936), the silicone oil would superwet the micropillar-arrayed silicon surface. Since the capillary length for silicone oil is $l_{C A}=\sqrt{\gamma_{L V} /(\rho g)} \approx 1.48 \mathrm{~mm}$, the ratio $R_{0} / l_{C A}$ is less than $17 \%$ (the droplet radius $R_{0}$ is about $0.25 \mathrm{~mm}$ ), which makes the surface tension take priority over gravity in the wetting process. The droplet was deposited on the micropillararrayed surface using a micropipette. The liquid motion was captured using a highmagnification microscope (DZ3, Union) and a high-speed camera (HotShot $512 \mathrm{sc,}$ 


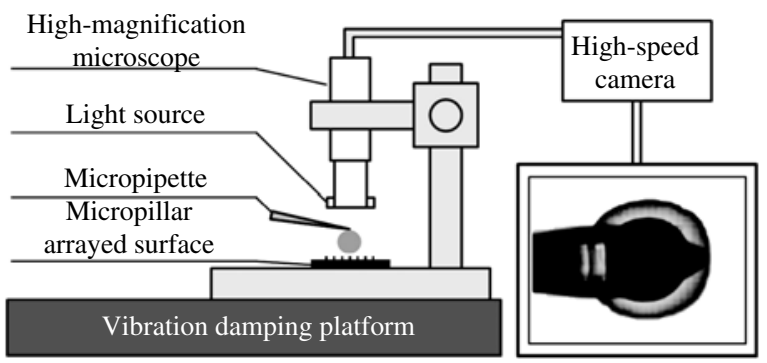

FIGURE 2. A schematic of the experimental setup.

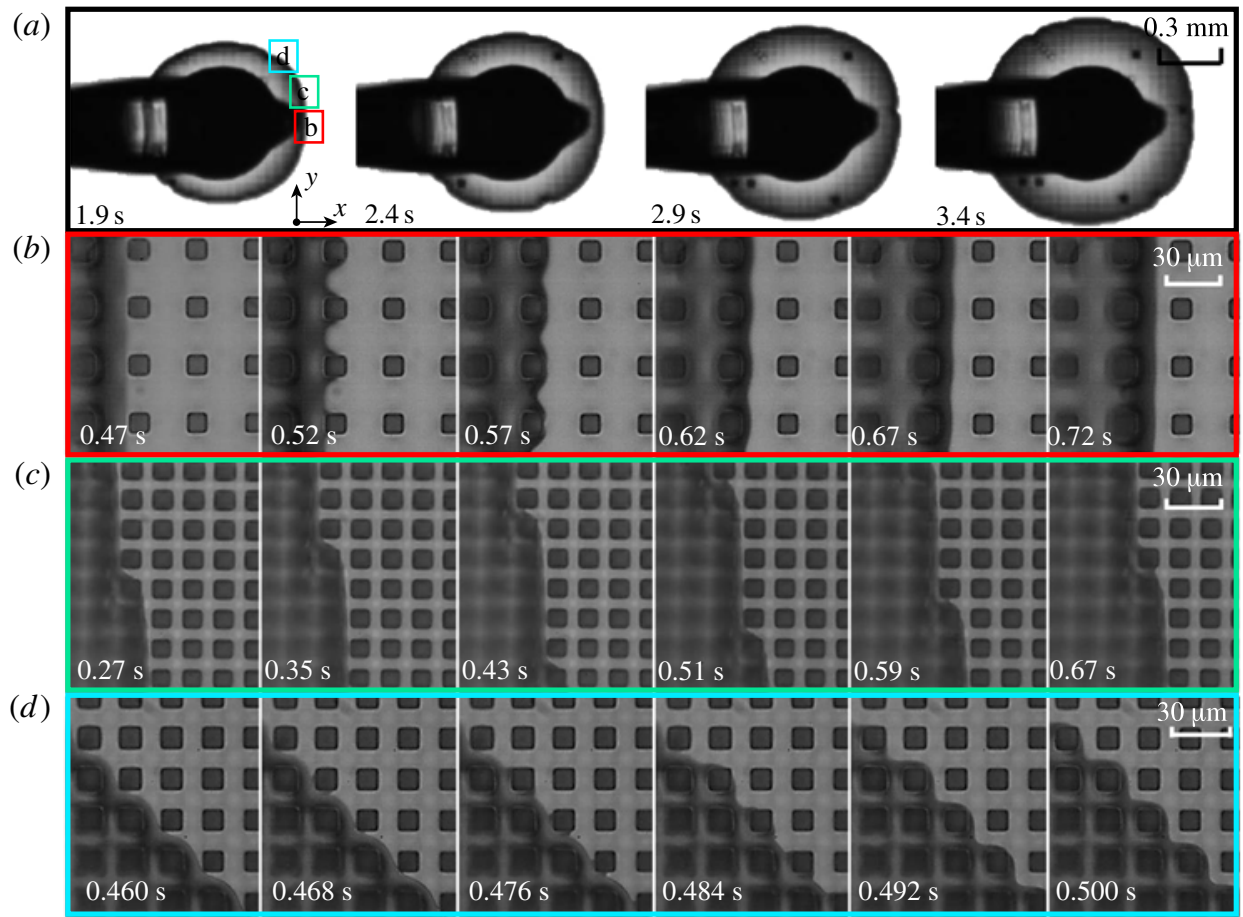

FIGURE 3. (Colour online) (a) Snapshots of the whole droplet under an optical microscope, $\Delta t=0.5 \mathrm{~s}$. $(b-d)$ Snapshots of different parts of the droplet labelled in $(a)$, under a highmagnification microscope and high-speed camera, (b) $\Delta t=50 \mathrm{~ms}$, ro $=1.42$ (sample 6, table 1); (c) $\Delta t=80 \mathrm{~ms}$, $r o=2.49$ (sample 2, table 1); $(d) \Delta t=8 \mathrm{~ms}$, ro $=1.94$ (sample 4 , table 1).

NAC) at a frame rate up to 5000 fps. A schematic of the experimental setup is shown in figure 2.

When the droplet was deposited on the pillar-arrayed lyophilic surface, the droplet rapidly spread and could be divided into two parts: the bulk and the fringe (figures 1 and 3). The MCL advanced with a characteristic capillary velocity of $U_{C A} \sim \gamma_{L V} / \mu \sim 0.1 \mathrm{~m} \mathrm{~s}^{-1}$. The fringe propagated much faster than the bulk $\left(R_{f}>R_{b}\right)$ due to the driving force induced by the excess lyophilic surface. Under low-magnification observation, the outline of the fringe was a circle, as shown in 

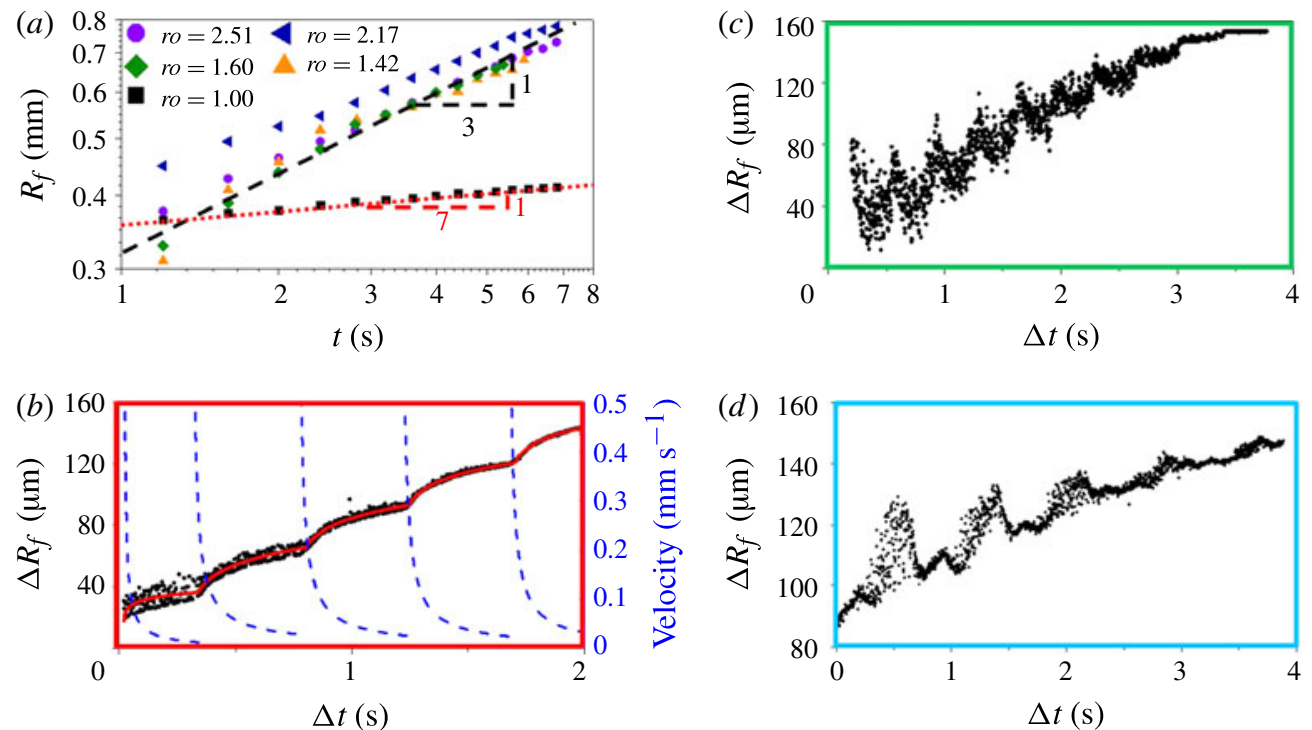

FIgure 4. (Colour online) Experiments. (a) Propagation of the fringe radius with respect to time on different rough substrates, as listed in table 1 . The dotted red and dashed black lines represent the scaling laws $R \sim t^{1 / 7}$ and $R \sim t^{1 / 3}$, respectively. $(b-d)$ Propagation of the fringe radius with respect to time corresponding to figure $2(b-d)$, respectively. In $(b)$, the red line is a fitted line using the power law, while the dashed blue line is a differential to the red line.

figure 3(a). The corresponding $R_{f}-t$ plots for different samples (table 1) are shown in figure $4(a)$. Obviously, the propagation of the droplet on a rough surface (colour points) and a smooth surface (black points) followed different scaling laws. Although with different speeds depending on $r o(>1)$, the fringe approximately obeyed a scaling law of $R_{f} \sim t^{1 / 3}$. Meanwhile, the radius of a droplet on a smooth surface $(r o=1)$ obeyed a scaling law of $R \sim t^{1 / 7}$, which was in agreement with previous experiments (Fetzer et al. 2009) and simulations (De Coninck \& Blake 2008). The micropillar array accelerated the wetting process, causing the lyophilic solid surface to become superlyophilic. Observed under the microscope, the dark area means there is an interface inclined with the substrate, while the bright area means the interface is parallel to the substrate.

Under a high-magnification microscope, the propagation of different parts of the fringe was quite different. In figure $3(b)$, the fringe advanced in the $x$ direction. The MCL was initially $(\Delta t=0.47 \mathrm{~s}$, figure $3 b)$ linear on the substrate at a velocity $U \sim 0.01 \mathrm{~mm} \mathrm{~s}^{-1}$ (dashed blue line, figure $4 b$ ). Once the MCL reached the pillars $(\Delta t=0.52 \mathrm{~s}$, figure $3 b)$, the excess driving force forced the MCL to accelerate, making the liquid propagate much faster at the interior corner between the pillar and the substrate, known as the Concus-Finn effect (Concus \& Finn 1969). In an interior corner with opening angle $2 \alpha$, the equilibrium velocity could be calculated as $U=f U_{C A}=f \gamma_{L V} / \mu$ (Weislogel \& Lichter 1998), where $f=\sin \alpha\left(\cos \theta_{0}-\sin \alpha\right) / S$ is the topological coefficient for capillary flow at the interior corner. $\theta_{0}$ is the equilibrium contact angle. $S$ is the slenderness ratio of the interior corner, i.e. the ratio of the length to the height of the liquid in the interior corner $(L / H$ in Weislogel \& Lichter (1998, figure 3)). So, the initial excess velocity could be 
estimated as

$$
U_{e x}=\frac{\gamma_{L V}}{S \mu}\left[\sin \alpha_{1}\left(\cos \theta_{0}-\sin \alpha_{1}\right)-\sin \alpha_{2}\left(\cos \theta_{0}-\sin \alpha_{2}\right)\right] .
$$

Substituting the experimental parameters into (2.1) $\left(\alpha_{1}=45^{\circ}\right.$ is the interior angle between the pillars and the substrate, $\alpha_{1}=90^{\circ}$ for the flat substrate, $\theta_{0}=0^{\circ}, S \sim 10$ ), the initial excess $U_{e x}$ is of the order of $1 \mathrm{~mm} \mathrm{~s}^{-1}$. Because the slow flow on the flat substrate restrained the fast flow at the interior corner, the average velocity was about $0.1 \mathrm{~mm} \mathrm{~s}^{-1}$, as shown in figure $4(b)$. The fast and slow part of the fringe reached a dynamic balance $(\Delta t=0.57 \mathrm{~s})$. Then, the liquid was pinned around the pillars $(\Delta t=0.62 \mathrm{~s}$ in figure $3 b$ ) and did not advance until the liquid between the two pillars caught up. After the MCL passed the pillars $(\Delta t=0.67 \mathrm{~s}$ in figure $3 b)$, the top of the pillars became blurry, which implied that the fringe climbed up the pillars and covered the top. The front of the fringe is dark owing to the inclined liquid-vapour interface. The MCL gradually decelerated to $U \sim 0.01 \mathrm{~mm} \mathrm{~s}^{-1}$ and spread again in a straight line $(\Delta t=0.72 \mathrm{~s}$ in figure $3 b)$ on the substrate driven only by the interface energy. In this process, the area between the first and second rows of micropillars gradually became brighter, implying that the liquid had filled the space between the pillars and gradually made the liquid-vapour interface parallel to the substrate. Figure $3(b)$ corresponds to the black points during $\Delta t \in[0.47,0.72] \mathrm{s}$ in figure $4(b)$. This process repeated during every period of the pillars. And the average velocity of the fringe gradually decreased because of the energy dissipations in the flow and in the three-phase zone, in accordance with that in figure 4(a), as will be discussed in $\S 4$.

In figures $3(c)$ and $4(c)$ the angle between the fringe velocity and the $x$ direction was about $12^{\circ}$, while in figures $3(d)$ and $4(d)$ it is about $44^{\circ}$. So unlike figure $3(b)$, the liquid encountered the pillars in sequence. The fringe would also be accelerated (Seemann et al. 2005) and pinned around the pillar one after another, making the MCL a complex 'zipping' line (Courbin et al. 2007). The progress was also periodically repeated. The zigzag lines at microscopic length scales formed a circle when observed in low magnification. The high-magnification microscope and high-speed camera provide us with more details of the propagation of the fringe and MCL. However, to observe how the PF develops into a fringe and obtain the flow fields at atomic level, MD simulations would not only provide us with a powerful tool to obtain detailed information in the entire wetting process, but also be a good supplement to the experiments to assist us in understanding the dynamic wetting process.

In the dynamic spreading process of a droplet on the pillar-arrayed surface, the surface tension $\gamma_{L V}$, the viscosity $\mu$ and the inertia govern the liquid motions. For the liquid, the ratio of viscous dissipation to surface tension and inertia is characterized by a dimensionless number, the Ohnesorge number $O h=\mu / \sqrt{\rho \gamma_{L V} L}$, where $\rho$ and $L$ are the density and characteristic length scale (we adopt $L$ to be the period of the pillars $p)$, respectively. In the experiments, $O h \sim 1\left(\rho=980 \mathrm{~kg} \mathrm{~m}^{-3}, \mu=0.091 \mathrm{~Pa} \mathrm{~s}\right.$, $\gamma_{L V}=0.021 \mathrm{~N} \mathrm{~m}^{-1}$ and $L \sim 10 \mu \mathrm{m}$ for the silicone oil droplet at $20^{\circ}$ and $1 \mathrm{bar}$ ), the fringe advanced $L \sim 1 \mathrm{~mm}$ during several seconds. In order to execute MD simulations that are geometrically and physically similar to the experiments, a liquid-solid pair should be carefully chosen so that the fringe propagates $L \sim 1 \mathrm{~nm}$ during several nanoseconds. We choose the liquid to be water using the extended simple point charge (SPC/E) water model (Berendsen, Grigera \& Straatsma 1987) with $\mu=0.729 \mathrm{mPa} \mathrm{s}$ (González \& Abascal 2010), $\rho=994 \mathrm{~kg} \mathrm{~m}^{-3}, \gamma_{L V}=0.0636 \mathrm{~N} \mathrm{~m}^{-1}$, (Vega \& De Miguel 2007), and $L \sim 1 \mathrm{~nm}$ for the SPC/E water droplet at $300 \mathrm{~K}$ and 1 bar. Consequently $O h \sim 1$ in the MD simulations, the same order as $O h$ in the experiments. 



FIgURE 5. (Colour online) $(a-h)$ MD simulations of a droplet on the pillar-arrayed hydrophilic surface ( $r o=1.63$, sample 3 , table 2 ) from $0.0-2.8 \mathrm{~ns} ;(1-8)$ side views of $(a-h)$. The dark grey and light grey balls are atoms of the substrate and the pillars, respectively. The surface of the droplets is shown in cyan.

In this way, the dynamic motion of the liquid is physically similar in the experiments and MD simulations. For the solid substrate, the roughness of the substrate ranges from 1.00 to 2.51 in both the experiments and MD simulations. The topologies of the pillar arrays were chosen to be geometrically similar in the experiments and MD simulations.

\section{Molecular dynamics simulations}

Large-scale MD simulations implemented in LAMMPS (Plimpton 1995) were carried out under a constant temperature of $300 \mathrm{~K}$ to explore the dynamic wetting process of a droplet on a nanopillar-arrayed lyophilic surface at the atomic level. The simulation domain is shown in figure $5(a)$ : the simulation box is $16.3 \mathrm{~nm} \times 16.3 \mathrm{~nm} \times$ $20.0 \mathrm{~nm}$. The rough substrate is a hydrophilic solid surface with a topography based on pillars. In the SPC/E water model, the oxygen atoms were modelled as charged Lennard-Jones $(\mathrm{LJ})$ particles $\left(\sigma_{O-O}=0.3166 \mathrm{~nm}, \varepsilon_{O-O}=0.650 \mathrm{~kJ} \mathrm{~mol}^{-1}\right.$, $q_{O}=-0.8476 \mathrm{e}$ ), while the hydrogen atoms were charged but without considering LJ interactions between them $\left(\sigma_{H-H}=0.0 \mathrm{~nm}, \varepsilon_{H-H}=0.0 \mathrm{~kJ} \mathrm{~mol}^{-1}, q_{H}=0.4238 \mathrm{e}\right)$. The SPC/E model is a slight reparametrization of the simple point charge (SPC) model, with a modified value of $q_{O}$ (charge on oxygen atoms) and $q_{H}$ (charge on hydrogen atoms), in order to add an average polarization correction to the potential energy function. The properties of the SPC/E water model have been well studied with $\mu=0.729 \mathrm{mPa}$ s (González \& Abascal 2010), $\rho=994 \mathrm{~kg} \mathrm{~m}^{-3}, \gamma_{L V}=0.0636 \mathrm{~N} \mathrm{~m}^{-1}$, (Vega \& De Miguel 2007) at $300 \mathrm{~K}$ and 1 bar, which are very close to those of the real bulk water. The solid atoms were modelled as uncharged LJ particles with $\sigma_{S-S}=0.2637 \mathrm{~nm}$ and $\varepsilon_{S-S}=42.5723 \mathrm{~kJ} \mathrm{~mol}^{-1}$, which were chosen to be hydrophilic to the SPC/E water. The values of $\sigma$ and $\varepsilon$ between them were calculated according to the Lorentz-Berthelot rule, $\sigma_{x-y}=\left(\sigma_{x-x}+\sigma_{y-y}\right) / 2$ and $\varepsilon_{x-y}=\left(\varepsilon_{x-x} \times \varepsilon_{y-y}\right)^{1 / 2}$, 


$\begin{array}{lccccccc}\text { Sample } & 1 & 2 & 3 & 4 & 5 & 6 & \text { Smooth } \\ d(\mathrm{~nm}) & 1.0195 & 2.0390 & 1.6312 & 2.0390 & 2.4468 & 3.0585 & \\ p(\mathrm{~nm}) & 2.0390 & 3.0585 & 3.2624 & 4.0480 & 4.8936 & 6.1170 & \\ h(\mathrm{~nm}) & 1.0195 & 1.0195 & 1.0195 & 1.0195 & 1.0195 & 1.0195 & \\ \varphi_{s}=\frac{d^{2}}{p^{2}} & 0.2500 & 0.4444 & 0.2500 & 0.2537 & 0.2500 & 0.2500 & \\ r o=1+\frac{4 d h}{p^{2}} & 2.00 & 1.89 & 1.63 & 1.50 & 1.42 & 1.33 & 1.0000\end{array}$

TABLE 2. Substrates of different roughness used in the MD simulations.

where the subscripts $x$ and $y$ represent the types of atoms. So $\sigma_{O-H}=0.1583 \mathrm{~nm}$, $\sigma_{S-H}=0.1319 \mathrm{~nm}, \sigma_{S-O}=0.2902 \mathrm{~nm}$ and $\varepsilon_{O-H}=0.0 \mathrm{~kJ} \mathrm{~mol}^{-1}, \varepsilon_{S-H}=0.0 \mathrm{~kJ} \mathrm{~mol}^{-1}$, $\varepsilon_{S-O}=5.2604 \mathrm{~kJ} \mathrm{~mol}^{-1}$. The $\mathrm{LJ}$ interactions between hydrogen and oxygen atoms, as well as between hydrogen and solid atoms, were ignored. The total potential energy $E_{i j}$ between two atoms $i$ and $j$ separated by $r_{i j}$ is the sum of the LJ potential energy and Coulombic pairwise interaction,

$$
E_{i j}=4 \varepsilon_{i j}\left[\left(\frac{\sigma_{i j}}{r_{i j}}\right)^{12}-\left(\frac{\sigma_{i j}}{r_{i j}}\right)^{6}\right]+k_{e} \frac{q_{i} q_{j}}{r_{i j}},
$$

where $\varepsilon$ is the depth of the potential well, $\sigma$ is the zero-crossing distance for the potential, $k_{e}=8.988 \times 10^{9} \mathrm{~N} \mathrm{~m}^{2} \mathrm{C}^{-2}$ is the Coulomb constant, and $q$ is the charge on the atom. The cut-off length for both LJ potential and Coulombic interaction is $1 \mathrm{~nm}$. The NVT ensemble (constant number of atoms, volume and temperature) was used. The Nosé-Hoover thermostat, acting as a heat reservoir, with a time step of 1 fs was employed to regulate the temperature at $300 \mathrm{~K}$. The water droplet with diameter of $6 \mathrm{~nm}$ was placed on a hydrophilic surface with pillars of different sizes. The topological parameters $[d, p, h]$ for simulated samples are listed in table 2 . The evaporated water molecules quickly saturate the simulation box, so the evaporation effect on the wetting process can be ignored.

In the MD simulations, the PF was defined to be a single layer of water molecules in the leading edge of the droplet. As shown in figure 5, when the droplet was placed on the substrate, both the PF and the bulk droplet propagated (Wang et al. 2009). The $\mathrm{PF}$ advanced more quickly than the bulk droplet, as illustrated in figure 6(a). Once the PF came into contact with the pillars, it accelerated, especially at the interior corner between the pillar and the substrate, shown in figure $6(d)$. Because of the confinement, the potential surface at the interior corner is lower and smoother than that on the solid surface (Yuan \& Zhao 2012). Hence a confined liquid molecule chain propagating ahead of the bulk droplet, i.e. a precursor chain, at the interior corner are more stable, possess less friction, and propagate faster than the PF with respect to the interior angle. Moreover, owing to the lower potential at the interior corner, the liquid molecules were pinned around the pillars (figure $6 e$ ), the PF climbed up to the top of the pillars (figure $6 b$ ), and covered the pillar surface. Then, the fringe of the droplet advanced, based on the PF, and filled the space between pillars, as illustrated in figures 5 and 6 . The bulk droplet propagated on the base of the PF and the fringe. The radius of the wet area expanded.

The variations of average $R_{f}, R_{b}$ and contact angle $\theta$ of the bulk droplet with respect to $t$ for different ro are plotted in figure 7. Obviously, the propagation of 
(a)

(b)
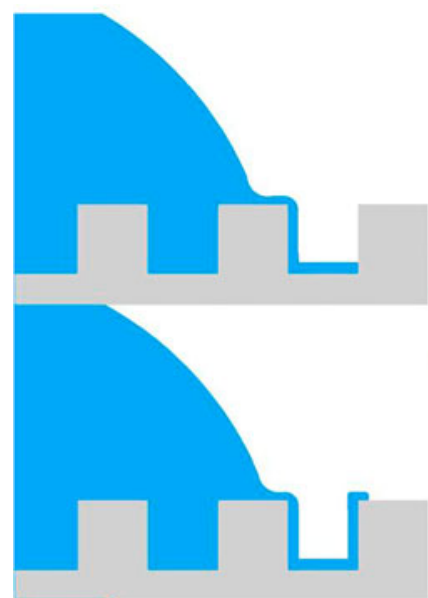

(c)

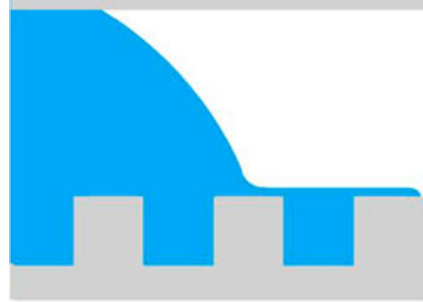

$(d)$



(e)

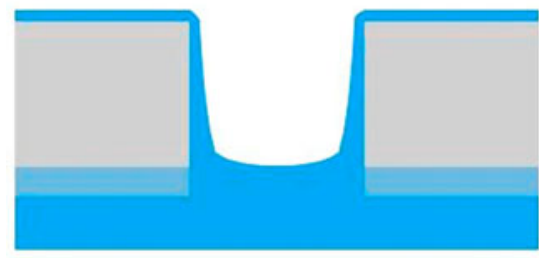

$(f)$

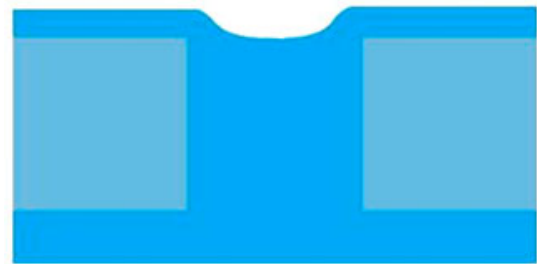

FIGURE 6. (Colour online) Schematic of $(a)$ the precursor film (PF) reaching the pillars, $(b)$ the PF climbing up the pillars, $(c)$ the fringe filling the space between pillars, $(d)$ the precursor chain (PC) along the interior corner, $(e)$ the PC being pinned around the pillars, $(f)$ the fringe filling the space between pillars. $(a-c)$ Side view, $(d-f)$ top view. The dark (blue) and light (grey) regions represent the liquid and the solid, respectively.

the droplet on a smooth surface (black squares) and a pillar-arrayed surface (colour squares) followed different scaling laws. The radius of a droplet on a smooth surface ( $r o=1$ ) obeyed a scaling law of $R \sim t^{1 / 7}$, in agreement with previous experiments (Fetzer et al. 2009) and simulations (De Coninck \& Blake 2008). Meanwhile, although with different speed depending on $r o(>1)$, the fringe approximately obeyed a scaling law of $R_{f} \sim t^{1 / 3}$. The propagation velocity increased with the increase in roughness, as discussed in $\S 4$ below. If the smooth surface changed to a pillar-arrayed surface, the scaling exponent would greatly change from about $1 / 7$ to about $1 / 3$. The topology enhances the wettability, accelerates the propagation, and causes the initial hydrophilic surface to become superhydrophilic. What is the physical mechanism of this transition? In figure $7(a, b)$ we can find that although $R_{b}$ expanded more slowly than $R_{f}$, they scaled similarly, which is also validated by the experiments (Kim et al. 2011). According to the MD simulations, we would assume $\alpha R_{b} \sim R_{f} \sim t^{n}$ in the next section.

\section{Scaling analysis using molecular kinetic theory}

We adopted MKT, which was proposed by Glasstone, Laidler \& Eyring (1941) and developed by Blake \& De Coninck (2002), to obtain the scaling law when a droplet propagates on a lyophilic rough surface (figure 8). According to the viewpoint of MKT, the wetting process is a stress-modified molecular rate process. The motion of the MCL is determined by the statistical dynamics of the molecules at the MCL. 

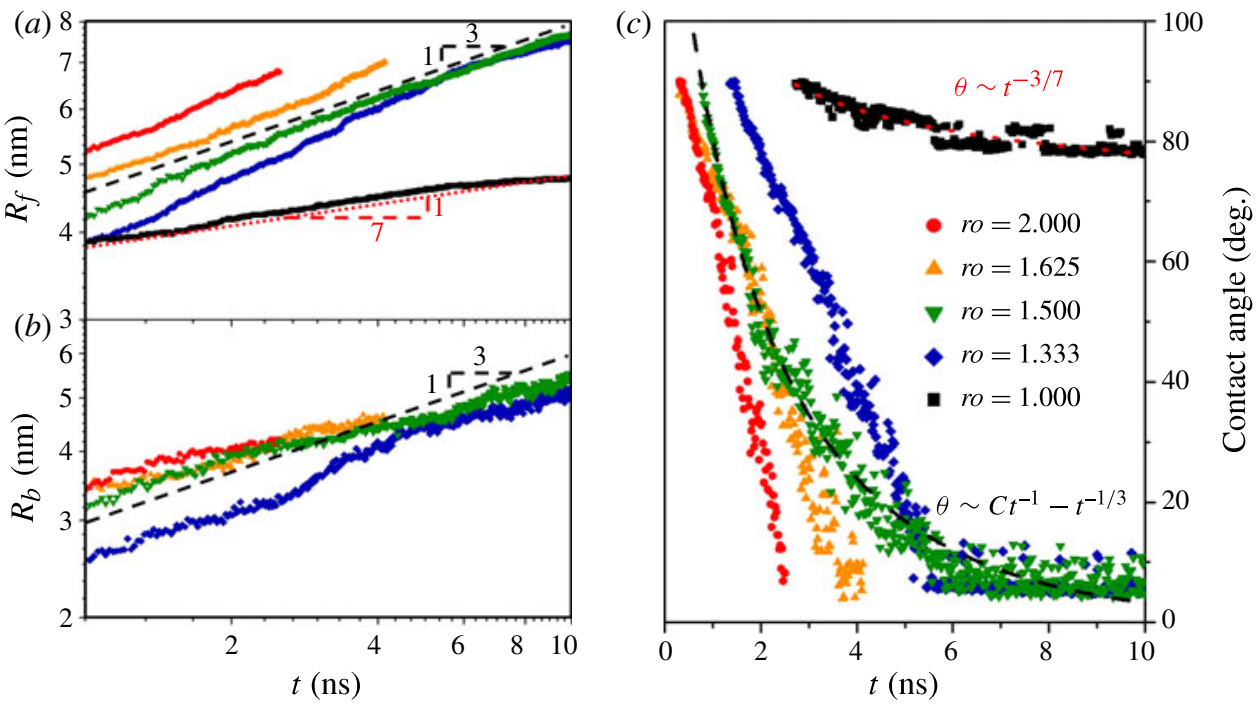

Figure 7. (Colour online) Simulations. Propagation of $(a)$ the fringe radius and $(b)$ the bulk-droplet radius with respect to time. The dotted red and dashed black lines represent the scaling laws $R \sim t^{1 / 7}$ and $R \sim t^{1 / 3}$, respectively. (c) Development of the contact angle with respect to time. To avoid covering the green points, the rest of the red and orange points are not plotted. The dotted red and dashed black lines represent the scaling laws $\theta \sim t^{-3 / 7}$ and $\theta \sim C t^{-1}-t^{-1 / 3}$, respectively.

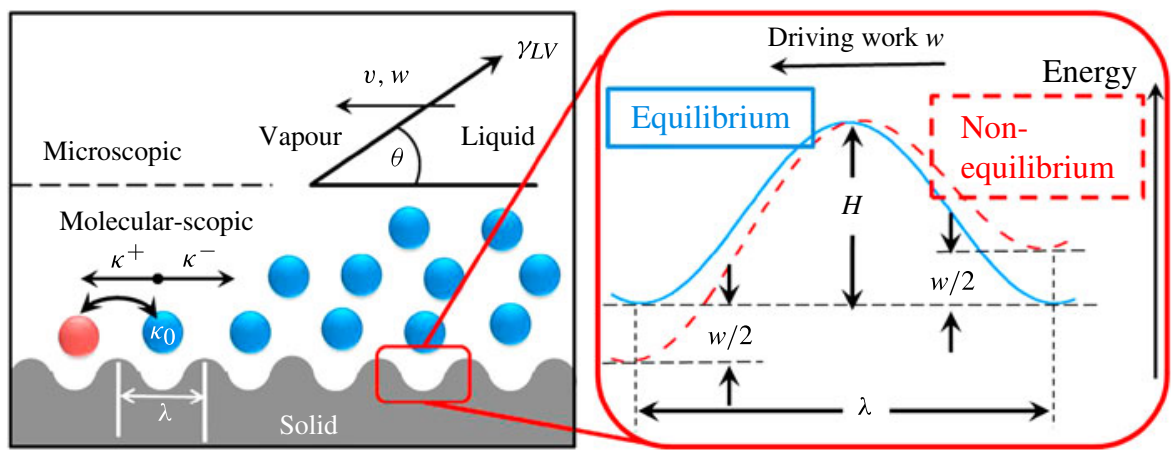

FIGURE 8. (Colour online) Schematic representation of the molecular kinetic model of wetting.

In fact, when the liquid moves across a solid surface, the solid adsorbs and tends to immobilize the liquid molecules, while the liquid molecules desorb and tend to advance. Considering the equilibrium liquid molecules in the vicinity of a solid, their advancing frequency $\kappa^{+}$and receding frequency $\kappa^{-}$are the same: $\kappa^{+}=\kappa^{-}=\kappa_{0}$, where $\kappa_{0}$ is the equilibrium frequency and can be expressed in terms of the activation energy $\Delta G$,

$$
\kappa_{0}=\frac{k_{B} T}{h} \exp \left(-\frac{\Delta G}{N_{A} k_{B} T}\right),
$$


where $k_{B}, T, h$ and $N_{A}$ are the Boltzmann constant, absolute temperature, the Planck constant and Avogadro number, respectively. In the case of liquid molecules adsorbing on a solid substrate, the activation energy $\Delta G$ is related to the work of adhesion between the solid and the liquid $W a$ and the spacing between surface sites $\lambda$. Hence, we could obtain $\kappa_{0}=\left(k_{B} T / \mu v_{m}\right) \exp \left(-\lambda^{2} W a / k_{B} T\right)$, where $v_{m}$ and $\mu$ are the molecular flow volume and fluid viscosity, respectively (Blake \& De Coninck 2002). Once the materials and topology of the liquid-solid interface are confirmed, $\kappa_{0}$ is a constant.

When a driving force is applied to the liquid molecules, the equilibrium is disturbed. The advancing and receding frequencies are no longer in balance, and the contact line moves relative to the solid. Under the driving work per unit area $w$, the corresponding frequencies are

$$
\begin{gathered}
\kappa^{+}=\kappa_{0} \exp \left(\frac{w \lambda^{2}}{2 k_{B} T}\right), \\
\kappa^{-}=\kappa_{0} \exp \left(-\frac{w \lambda^{2}}{2 k_{B} T}\right) .
\end{gathered}
$$

The driving force tilts the potential surface and makes it more difficult for molecules to move in the opposite direction (figure 8). The resulting velocity of the MCL can be expressed by

$$
U=\left(\kappa^{+}-\kappa^{-}\right) \lambda=2 \frac{k_{B} T \lambda}{\mu v_{m}} \exp \left(-\frac{\lambda^{2} W a}{k_{B} T}\right) \sinh \frac{w \lambda^{2}}{2 k_{B} T} .
$$

In our case, the ratio of the driving work $w \lambda^{2}$ to the thermal energy $k_{B} T$ is of the order of $0.1-1 ; \sinh w \lambda^{2} / 2 k_{B} T \sim w \lambda^{2} / 2 k_{B} T$ would be a good approximation to simplify (4.4) to $U \sim\left(w \lambda^{3} / \mu v_{m}\right) \exp \left(-\lambda^{2} W a / k_{B} T\right)$. Once the liquid-solid pair and the topology of the substrate are fixed, $\mu, \lambda, v_{m}, W a$ are all constant, $U \sim w / \mu$.

The driving work per unit area $w$ is equal to the total change of interface energy when the droplet propagates, $w=\left(\gamma_{S V}-\gamma_{S L}\right) \cdot r o-\gamma_{L V} \cos \theta$, where $\gamma_{S L}, \gamma_{S V}$ and $\gamma_{L V}$ are the solid-liquid, solid-vapour and liquid-vapour interface energies, respectively, and $\theta$ is the instant contact angle. Taking account of Young's equation $\left(\gamma_{S V}-\gamma_{S L}=\gamma_{L V} \cos \theta_{0}\right.$, $\theta_{0}$ is the static contact angle) (Young 1805), $\left(\gamma_{L V} / \mu\right) r o \cdot \cos \theta_{0}-U \sim\left(\gamma_{L V} / \mu\right) \cos \theta$. Considering the lubrication approximation $\left(H_{b} \ll R_{b}, \theta \sim H_{b} / R_{b} \sim 0\right)$ (Greenspan 1978), $U \sim \gamma_{L V} \theta^{2} / \mu$. The droplet spreads at the characteristic capillary velocity $\left(\gamma_{L V} / \mu\right)$, until it reaches an equilibrium state $(U \rightarrow 0)$. In the equilibrium state, for a rough surface $(r o>1)$ we obtain Wenzel's relation, $\cos \theta=r o \cdot \cos \theta_{0}\left(\theta<\theta_{0}\right)$ (Wenzel 1936); for a smooth surface ( $r o=1$ ) we get Young's equation (Young 1805).

When the droplet wets the substrate, the initial spherical droplet with radius $R_{0}$ evolves into the bulk droplet and the fringe. Since the liquid is a non-volatile liquid, the volume remains constant, $V_{0}=V_{\text {bulk }}+V_{\text {fringe }}$, i.e. $4 \pi R_{0}^{3} / 3=\left(\pi H_{b}\left(3 R_{b}^{2}+H_{b}^{2}\right)+\right.$ $\left.\pi\left(1-\varphi_{s}\right) h R_{f}^{2}\right) / 6$, where $R_{f}$ is the average radius of the fringe and $\varphi_{s}$ is the density of roughness ( $\varphi_{s}=d^{2} / p^{2}$ for pillars). In the spreading process, $\alpha R_{b} \sim R_{f} \sim R$ is validated by Kim et al. (2011) and our MD simulations, where $\alpha(\alpha \leqslant 1)$ is independent of time. So $\theta \sim H_{b} / R_{b} \sim\left(8 R_{0}^{3}-6\left(1-\varphi_{s}\right) h R_{f}^{2}\right) / 3 R_{b}^{3} \sim\left(8 R_{0}^{3}-6\left(1-\varphi_{s}\right) h R^{2}\right) / 3 R^{3}$. This leads to

$$
U=\frac{\mathrm{d} R}{\mathrm{~d} t} \sim \frac{\gamma_{L V}}{\mu}\left(\frac{4 R_{0}^{3}}{R^{3}}-\frac{3 \bar{h}}{R}\right)^{2},
$$

where $\bar{h}=\left(1-\varphi_{s}\right) h$ is the effective height of the pillars. According to (4.5), we can define a characteristic length $L=\sqrt{\left(4 \pi R_{0}^{3} / 3\right) /\left(\pi\left(1-\varphi_{s}\right) h\right)} \sim R_{0} \sqrt{R_{0} / \bar{h}}$, which 
represents the maximum propagation radius of the fringe and also determines whether the effect of surface roughness should be considered. Hence, two extreme regimes can be distinguished. One is for the smooth surface and the other is for the rough surface.

(i) When $L \gg R_{f}$, i.e. relatively large droplet, short or sparse pillars, the effect of surface roughness can be neglected. Here $U \sim \gamma_{L V} R_{0}^{6} / \mu R^{6}$. The dimensionless solution is $R / R_{0} \sim\left(t / \tau_{c}\right)^{1 / 7}$. The characteristic time $\tau_{c}=\mu R_{0} / \gamma_{L V}$ for the droplet to spread by $R_{0}$ with capillary velocity $U_{C A}=\gamma_{L V} / \mu$ is only controlled by the characteristic properties of the bulk liquid, i.e. $\gamma_{L V} / \mu$ and $R_{0}$. The scaling law of a droplet spreading on a smooth solid surface is $R / R_{0} \sim\left(t / \tau_{c}\right)^{1 / 7}$, which is confirmed by previous references (Blake 2006; Yuan \& Zhao 2010), our experiments (black squares in figure $4 a$ ) and our simulations (black squares in figure $7 a$ ). The contact angle $\theta \sim\left(R_{0} / R\right)^{3} \sim t^{-3 / 7}$, which is validated in figure $7(c)$.

(ii) When $L \ll R_{f}$, i.e. relatively small droplet, long or dense pillars, the effect of surface roughness dominates the dynamic wetting process. Here $U \sim \gamma_{L V} \bar{h}^{2} / \mu R^{2}$. The dimensionless solution is $R / R_{0} \sim\left(t / \tau_{c}\right)^{1 / 3}$, where the characteristic time $\tau_{c}=\mu R_{0}^{3} / \gamma_{L V} \bar{h}^{2}$ is controlled not only by properties of the bulk water, but also by the topological parameters of the rough surface: $\varphi_{s}$ and $h$. The contact angle $\theta$ of a droplet on a pillar-arrayed substrate $\theta \sim\left(8 R_{0}^{3}-6 \bar{h} R^{2}\right) / 3 R^{3} \sim C t^{-1}-t^{-1 / 3}$ ( $C$ is independent of time) decreased much faster than $\theta \sim\left(R_{0} / R\right)^{3} \sim t^{-3 / 7}$ for a droplet on a smooth substrate. When $\theta$ finally approaches $0^{\circ}, t \sim \mu R_{0}^{9 / 2} / \gamma_{L V} \bar{h}^{7 / 2}$ is of the order of $10 \mathrm{~s}$ in experiments or $1 \mathrm{~ns}$ in MD simulations, which is validated by experiments and simulations.

Expand the expression of velocity for case (ii), $U \sim\left[\gamma_{L V}\left(1-\varphi_{s}\right)^{2} h^{2} / \mu t^{2}\right]^{1 / 3}$. In our experiments and MD simulations, the pillar heights for different samples are nearly the same, so only the density of roughness $\varphi_{s}$ determines the time evolution of the radius and velocity. The smaller $\varphi_{s}$ is, the faster the liquid spreads on the substrate. In the experiments shown in figure $4(a)$, the orange points with the smallest $\varphi_{s}$ have the fastest velocity (steepest slope), while the blue points with the largest $\varphi_{s}$ have the slowest velocity (gentlest slope).

In our experiments, $R_{0} \sim 0.5 \mathrm{~mm}, h \sim 10 \mu \mathrm{m}$, and $\varphi_{s}=d^{2} / p^{2} \sim 10^{-1}$, so $L=$ $R_{0} \sqrt{R_{0} / \bar{h}} \sim 10^{-3} \mathrm{~m}<R_{f} \sim 10^{-2} \mathrm{~m}$. In our MD simulations, $R_{0} \sim 6 \mathrm{~nm}, h \sim 1 \mathrm{~nm}$, and $\varphi_{s}=d^{2} / p^{2} \sim 10^{-1}$, so $L=R_{0} \sqrt{R_{0} / \bar{h}} \sim 10^{-8} \mathrm{~m}<R_{f} \sim 10^{-7} \mathrm{~m}$. According to the above derivation, the scaling exponent of droplet on pillar-arrayed surfaces is approximately $1 / 3$, while the scaling exponent of droplet on a smooth surface is about $1 / 7$. Our scaling laws could be a starting point in understanding the behaviours and mechanisms of a droplet on lyophilic pillars. The regime between the two extreme regimes is complicated and requires further studies.

\section{Flow patterns and flow fields}

The above statistics in MD simulations and derivation of MKT are averaged for all directions of the droplet. However, the pillar-arrayed surface is anisotropic, and its wettability depends on the direction. In this section the main focus is the directiondependence of the evolution of $R_{f}$. So, the flow density and flow patterns for $r o=2$ and $r o=1.5$ are visualized in figure 9. The origin is put in the centre of the droplet. Because the centre of the droplet keeps moving, the figures are not symmetric with each other. Because the pillars are symmetric in the horizontal and vertical directions, the average outline of the fringe is almost a circle, labelled by the red lines in 
(a)

(b)

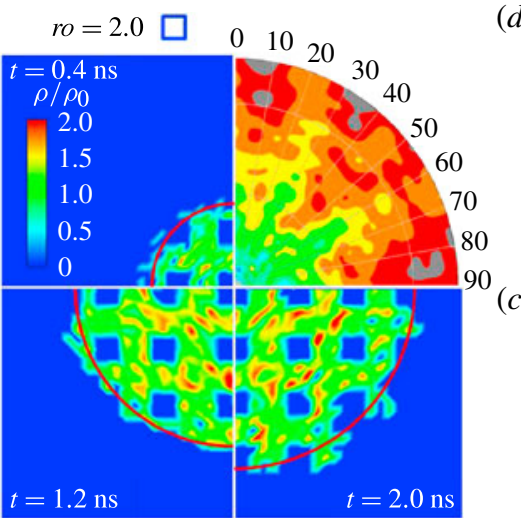

(d)

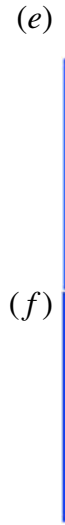

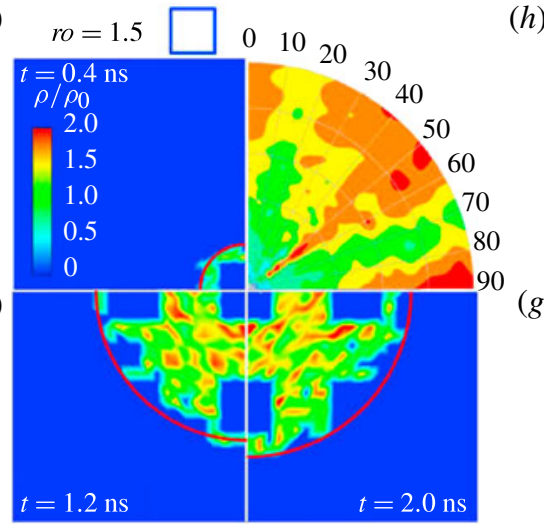

(h)



FIGURE 9. Flow densities and flow fields in MD simulations for different roughnesses: $(a-d)$ $r o=2.0$ (sample 1, table 2); $(e-h)$ ro $=1.5$ (sample 4, table 2). The blue squares represent the size and shape of the pillars. $(a-c, e-g)$ Sequence of snapshots of the propagation of the relative flow density $\rho / \rho_{0}$ in a top view, where $\rho_{0}=1000 \mathrm{~kg} \mathrm{~m}^{-3}$ is the density of bulk water. The inset colour legends in $(a, e)$ are for flow density. $(d, h)$ Propagation of the fringe radius with respect to time in polar coordinates. The origin $O$ is put in the centre of the droplet. The right-hand colour legend is for $(d, h)$. The radial coordinate represents time evolution, the angular coordinate represents the direction and the colour represents the distance between the origin and the fringe front. The total statistical time is $2.5 \mathrm{~ns}$.

figure $9(a-c, e-g)$. The average density is about of that of bulk water $\sim 10^{3} \mathrm{~kg} \mathrm{~m}^{-3}$. But still there are fluctuations in the liquid density, owing to the fluctuations of thermal energy at an instant moment.

However, owing to the topology of the pillars, the flow velocity depends on the direction, and there exist fast and slow directions for the liquid flow. Figure $9(d, h)$ is plotted in polar coordinates, in which the radial coordinate represents time evolution, the angular coordinate represents the direction and the colour represents the distance between the origin and the fringe front labelled by the right-hand colour legend. In figure $10(a)$, point $\mathrm{A}\left(1.5 \mathrm{~ns}, 20^{\circ}\right.$, yellow) represents the fringe at $20^{\circ}$ propagating about $48-56 \AA$ in $1.5 \mathrm{~ns}$. The line at $45^{\circ}$ in figure $10(a)$ represents propagation of the fringe at $45^{\circ}$ with respect to time in figure $10(b)$. So do the lines at $75^{\circ}$ and corresponding figure $10(c)$. Comparing figures $10(b)$ and $10(c)$, we find that the evolution of $R_{f}$ at $45^{\circ}$ is faster than that at $75^{\circ}$. However, the scaling law for $R_{f}$ at both 45 and $75^{\circ}$ is about $R_{f} \sim t^{1 / 3}$. As shown in figure $9(d, h)$, the fringe flowed faster in directions that are more hydrophilic (red colour), while the fringe flowed more slowly in directions that are less hydrophilic (green colour). The direction-dependence of the velocity creates a unique pattern for each given roughness. For $r o=2.0$, the fast directions were approximately 10,45 , and $80^{\circ}$, while the slow directions were about 30 and $60^{\circ}$. For $r o=1.5$, the fast directions were approximately 0,45 and $90^{\circ}$, while the slow directions were about 15 and $75^{\circ}$. For reasons of symmetric topology, the flow patterns were approximately symmetric about $45^{\circ}$. The fast and slow directions influenced each other, eventually reaching a dynamic balance, and made the outline of the fringe almost a circle. With the increase in roughness, the expanding velocity increased. The roughness in figure $9(a-d)$ was larger than that in figure $9(e-h)$, which made the pillar-arrayed surface more hydrophilic (more red colour). 
(a)

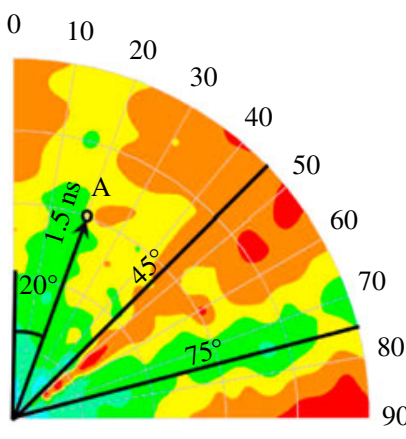

(b)

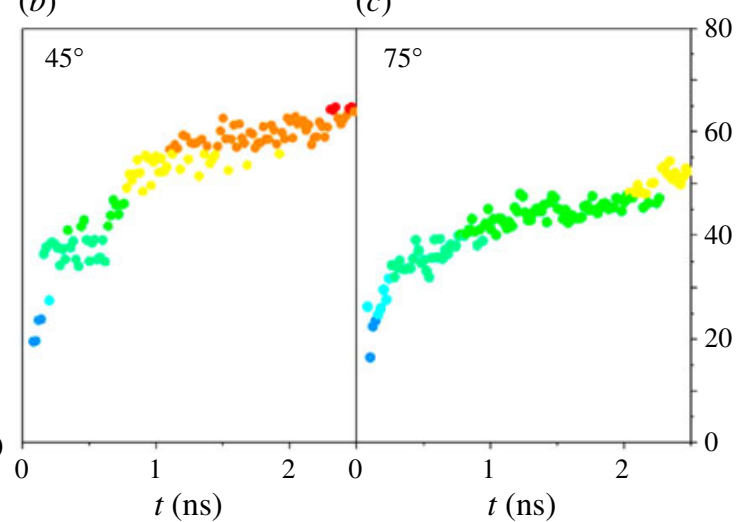

$R_{f}(\AA)$

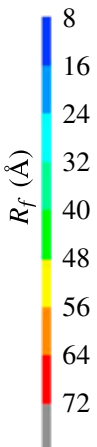

Figure 10. (a) Propagation of the fringe with respect to time in polar coordinates (ro=1.5). $(b-c)$ Propagation of fringe at 45 and $75^{\circ}$ labelled in $(a)$ with respect to time, respectively.

The pillars are square and ro was the same in both horizontal and vertical directions in figure 9. If rectangular pillars are used, i.e. $r o=1.5$ in the horizontal direction and $r o=2$ in the vertical direction, as shown in figure 11 $(a-d)$, the flow field will no longer be symmetric about $45^{\circ}$. In the vertical direction the flow field kept the characteristics in figure $9(d)$, while in the horizontal direction the flow field kept the characteristics in figure $9(h)$. Between the two directions, the flow field gradually changed, exhibiting a transition of wettability in figure $11(d)$. The rectangular pillars also drove the flow faster in the vertical direction than in the horizontal direction, which made the outline of the fringe an ellipse.

The flow patterns of liquid on different pillars are plotted together in figure 11(e). The transition of the flow pattern from $r o=2.0$ to $r o=1.5$ can be clearly observed. First, with the decrease in roughness, the area of red colour decreases and that of the green colour increases, which implies decrease in the average propagation velocity. The direction-dependence of the velocity gradually changed with the change of the roughness.

\section{Conclusions}

In this article, multiscale experiments and MD simulations have been used to explore the dynamic wetting process of a droplet on a pillar-arrayed lyophilic surface. The macroscopic radial expansion and the microscopic wetting of individual pillars have been investigated. Under a high-magnification microscope and a high-speed camera, the originally smooth and circular MCL has been found to propagate in a complex 'zipping' pattern depending on the propagation direction. The excess area of the rough surface imposes an excess driving force when the liquid reaches the pillars, and also pins the MCL until the slow part of the fringe catches up. The flow patterns and the flow fields of the droplet on the pillar-arrayed surface, influenced by the concerted effect of the liquid-solid interactions and the surface roughness, have been revealed from the continuum (experiments) to the atomic level (MD simulations). Based on results from multiscale experiments and MD simulations, the dynamic process and the physical mechanisms have been analysed theoretically using MKT. Controlled by the characteristic length scale $L$, the scaling laws for two regimes have 
(a)

(b)

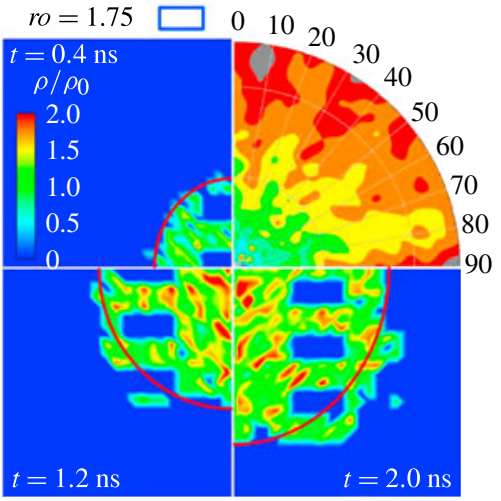

(d) $\quad(e)$

(c)

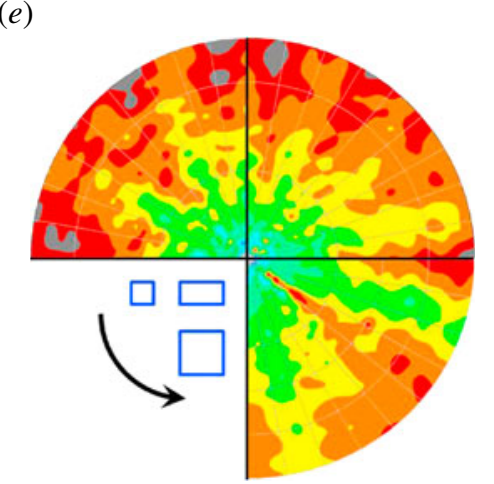

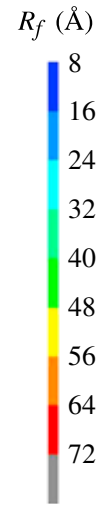

FIgURE 11. $(a-d)$ Flow densities and flow fields in MD simulations for rectangular pillars $(r o=1.75)$. The blue rectangles represent the size and shape of the pillars. $(a-c)$ Sequence of snapshots of the propagation of the relative flow density $\rho / \rho_{0}$ in a top view, where $\rho_{0}=1000 \mathrm{~kg} \mathrm{~m}^{-3}$ is the density of bulk water. The inset colour legend in $(a)$ is for flow density. $(d)$ Propagation of the fringe radius with respect to time in polar coordinates. $(e)$ Transition of flow patterns from $r o=2.0$ to $r o=1.5$. The origin $O$ is put in the centre of the droplet. The right-hand colour legend is for $(d)$ and $(e)$. The radial coordinate represents time evolution, the angular coordinate represents the direction and the colour represents the distance between the origin and the fringe front. The total statistical time is $2.5 \mathrm{~ns}$.

been obtained, one for the rough surface and the other for the smooth surface. Both experiments and simulations agree with the scaling law from MKT. Our results may help in understanding the dynamic wetting of the droplet on a pillar-arrayed lyophilic substrate and assisting the future design of pillar-arrayed lyophilic surfaces in practical applications.

\section{Acknowledgements}

This work was jointly supported by the National Natural Science Foundation of China (NSFC, Grant 11202213 and 11072244), the Key Research Program of the Chinese Academy of Sciences (Grant KJZD-EW-M01) and the Instrument Developing Project of the Chinese Academy of Sciences (Grant Y2010031).

\section{REFERENCES}

Berendsen, H. J. C., Grigera, J. R. \& Straatsma, T. P. 1987 The missing term in effective pair potentials. J. Phys. Chem. 91, 6269-6271.

Blake, T. D. 1993 Wettability. Dekker.

Blake, T. D. 2006 The physics of moving wetting lines. J. Colloid Interface Sci. 299, 1-13.

Blake, T. D. \& DE CONINCK, J. 2002 The influence of solid-liquid interactions on dynamic wetting. Adv. Colloid Interface Sci. 96, 21-36.

Bonn, D., Eggers, J., Indekeu, J., Meunier, J. \& Rolley, E. 2009 Wetting and spreading. Rev. Mod. Phys. 81, 739-805.

Concus, P. \& FinN, R. 1969 On the behaviour of a capillary surface in a wedge. Proc. Natl Acad. Sci. USA 63, 292-299.

Courbin, L., Denieul, E., Dressaire, E., Roper, M., Ajdari, A. \& Stone, H. A. 2007 Imbibition by polygonal spreading on microdecorated surfaces. Nat. Mater. 6, 661-664.

Cox, R. 1986 The dynamics of the spreading of liquids on a solid surface. Part 1. Viscous flow. J. Fluid Mech. 168, 169-194. 
De Coninck, J. \& Blake, T. D. 2008 Wetting and molecular dynamics simulations of simple liquids. Annu. Rev. Mater. Res. 38, 1-22.

Derjaguin, B. V., Churaev, N. V. \& Muller, V. M. 1987 Surface Forces. Consultants Bureau.

Dussan, V. E. B. 1976 The moving contact line: the slip boundary condition. J. Fluid Mech. 77, $665-684$.

DUSSAN, V. E. B. 1979 On the spreading of liquids on solid-surfaces: static and dynamic contact lines. Annu. Rev. Fluid Mech. 11, 371-400.

Fetzer, R., Ramiasa, M. \& Ralston, J. 2009 Dynamics of liquid-liquid displacement. Langmuir 25, 8069-8074.

FU, J., MAO, P. \& HAN, J. 2009 Continuous-flow bioseparation using microfabricated anisotropic nanofluidic sieving structures. Nat. Protoc. 4, 1681-1698.

De Gennes, P. G. 1985 Wetting: statics and dynamics. Rev. Mod. Phys. 57, 827-863.

Glasstone, S., Laidler, K. \& Eyring, H. 1941 The Theory of Rate Processes. McGraw-Hill.

GonzÁlez, M. A. \& AbAscal, J. L. F. 2010 The shear viscosity of rigid water models. J. Chem. Phys. 132, 096101.

Greenspan, H. 1978 On the motion of a small viscous droplet that wets a surface. J. Fluid Mech. 84, 125-143.

HАsimoto, H. 1959 On the periodic fundamental solutions of the Stokes equations and their application to viscous flow past a cubic array of spheres. J. Fluid Mech. 5, 317-328.

Hocking, L. 1976 A moving fluid interface on a rough surface. J. Fluid Mech. 76, 801-817.

HUH, C. \& SCRIVEN, L. 1971 Hydrodynamic model of steady movement of a solid/liquid/fluid contact line. J. Colloid Interface Sci. 35, 85-101.

Ishino, C., Reyssat, M., Reyssat, E., OKumura, K. \& Quéré, D. 2007 Wicking within forests of micropillars. Europhys. Lett. 79, 56005.

KARniAdAKIS, G., BEŞKÖK, A. \& AlurU, N. R. 2005 Microflows and Nanoflows: Fundamentals and Simulation. Springer.

Kim, S. J., Moon, M. W., Lee, K. R., Lee, D. Y., Chang, Y. S. \& KiM, H. Y. 2011 Liquid spreading on superhydrophilic micropillar arrays. J. Fluid Mech. 680, 477-487.

Mchale, G., Shirtcliffe, N., Aqil, S., Perry, C. \& Newton, M. 2004 Topography driven spreading. Phys. Rev. Lett. 93, 36102.

Nagrath, S., Sequist, L. V., Maheswaran, S., Bell, D. W., Irimia, D., Ulkus, L., Smith, M. R., KWAK, E. L., Digumarthy, S. \& Muzikansky, A. 2007 Isolation of rare circulating tumour cells in cancer patients by microchip technology. Nature 450, 1235-1239.

PArker, A. R. \& LAWrence, C. R. 2001 Water capture by a desert beetle. Nature 414, 33-34.

Plimpton, S. 1995 Fast parallel algorithms for short-range molecular-dynamics. J. Comput. Phys. 117, $1-19$.

QuÉRÉ, D. 2008 Wetting and roughness. Annu. Rev. Mater. Res. 38, 71-99.

REN, W. Q. \& E, W. N. 2007 Boundary conditions for the moving contact line problem. Phys. Fluids 19, 022101.

REN, W. Q., HU, D. \& E, W. N. 2010 Continuum models for the contact line problem. Phys. Fluids 22, 102103.

Seemann, R., Brinkmann, M., Kramer, E., Lange, F. \& Lipowsky, R. 2005 Wetting morphologies at microstructured surfaces. Proc. Natl Acad. Sci. USA 102, 1848-1852.

Skelley, A. M., Kirak, O., Suh, H., Jaenisch, R. \& Voldman, J. 2009 Microfluidic control of cell pairing and fusion. Nat. Meth. 6, 147-152.

Srivastava, N., Din, C., Judson, A., MacDonald, N. C. \& Meinhart, C. D. 2010 A unified scaling model for flow through a lattice of microfabricated posts. Lab on a Chip 10, 1148-1152.

TANner, L. 1979 The spreading of silicone oil drops on horizontal surfaces. J. Phys. D: Appl. Phys. 12, 1473-1484.

Teletzke, G. F., Davis, H. T. E. D. \& Scriven, L. 1987 How liquids spread on solids. Chem. Engng Commun. 55, 41-82.

VegA, C. \& DE Miguel, E. 2007 Surface tension of the most popular models of water by using the test-area simulation method. J. Chem. Phys. 126, 154707. 
Wang, C. L., Lu, H. J., Wang, Z. G., Xiu, P., Zhou, B., Zuo, G. H., Wan, R. Z., Hu, J. \& FANG, H. P. 2009 Stable liquid water droplet on a water monolayer formed at room temperature on ionic model substrates. Phys. Rev. Lett. 103, 137801.

Weislogel, M. M. \& Lichter, S. 1998 Capillary flow in an interior corner. J. Fluid Mech. 373, 349-378.

WENZEL, R. N. 1936 Resistance of solid surfaces to wetting by water. Ind. Engng Chem. 28, 988-994.

Xiao, R., Enright, R. \& WAng, E. N. 2010 Prediction and optimization of liquid propagation in micropillar arrays. Langmuir 26, 15070-15075.

Young, T. 1805 An essay on the cohesion of fluids. Phil. Trans. R. Soc. Lond. 95, 65-87.

YUAN, Q. Z. \& ZHAO, Y. P. 2010 Precursor film in dynamic wetting, electrowetting, and electro-elasto-capillarity. Phys. Rev. Lett. 104, 246101.

YUAN, Q. Z. \& ZHAO, Y. P. 2012 Topology-dominated dynamic wetting of the precursor chain in a hydrophilic interior corner. Proc. R. Soc. A 468, 310-322. 